\title{
Laboratory Biosafety Issues Related to Coronavirus Disease 2019
}

\begin{abstract}
Indroduction: The outbreak of Novel Coronavirus Pneumonia SARS-CoV-2 has necessitated the reinforcement of infection control measures in the hospital and laboratory setting. Contact and droplet infection control measures are advised for handling patients diagnosed with COVID-19 and airborne precautions for procedures that generate aerosols. Pre-ananalytical Phase: Risk assessment is conducted for all steps of laboratory processes viz. preanalytical, analytical and postanalytical. Standard Precautions must be followed at all times when laboratory staff handle clinical specimens that may contain infectious microorganism. Standard precautions must include hand hygiene along with the use of personal protective equipment (PPE). All samples are collected in appropriate containers and all containers are decontaminated by $62-71 \%$ ethanol (alcohol) before transporting them to the laboratory in triple packaging. Analytical Phase: All samples should undergo initial processing in a biosafety cabinet (BSC). It should be ensured while undertaking all technical procedures that there is minimal formation of aerosols and droplets. Post-analytical Phase: All biomedical waste should be disposed as per state and national guidelines. Decontamination includes use of $1 \%$ sodium hypochlorite, $62-71 \%$ ethanol for surface disinfection or Hydrogen peroxide $(0.5 \%)$. Conclusion: These laboratory biosafety measures are important to minimise the risk of laboratory transmission of COVID-19 to health care workers.
\end{abstract}

Keywords: Biosafety, coronavirus disease 2019, laboratory, personal protective equipment

\section{Introduction}

An outbreak of novel coronavirus pneumonia was first observed in Wuhan, China, late in the year 2019. ${ }^{[1]}$ The novel coronavirus disease has since been referred to as coronavirus disease 2019 (COVID-19) by the WHO. ${ }^{[2]}$ The outbreak was declared on January 30, 2020, by the WHO as a "public health emergency of international concern." Contact and droplet infection control measures are advised for handling patients diagnosed with COVID-19 and airborne precautions for procedures that generate aerosols by various health organizations such as WHO, the European Centre for Disease Prevention and Control, and the United States Centres for Diseases Control and Prevention (CDC).$^{[3-5]}$

In view of this risk of transmission associated with the virus, laboratory biosafety assumes great importance in handling laboratory specimens that are either suspected or confirmed for COVID-19 and utmost precautions should be taken regarding the same. CDC has defined biosafety as "the

This is an open access journal, and articles are distributed under the terms of the Creative Commons Attribution-NonCommercial-ShareAlike 4.0 License, which allows others to remix, tweak, and build upon the work non-commercially, as long as appropriate credit is given and the new creations are licensed under the identical terms.

For reprints contact: WKHLRPMedknow_reprints@wolterskluwer.com discipline addressing the safe handling and containment of infectious microorganisms and hazardous biological materials." ${ }^{\text {[6] }}$ One of the most important tools in this endeavor is risk assessment. Each laboratory makes this assessment based on their professional judgment and by following certain guidelines. This risk assessment should take into account the procedures undertaken at every step, competency level of the personnel who are performing these procedures, and availability of resources such as laboratory equipment and facility. The risk assessment is conducted for all steps of laboratory processes, viz., preanalytical, analytical, and postanalytical. All the processes are assessed for hazards such as spillage and aerosol formation and are graded accordingly ranging from very low to very high depending on the severity of the risk involved. Each institute creates its own guidelines with reference to national and international laid down criteria to mitigate these risks. The relevant staff is then trained for the same. The samples should be handled only by the trained staff.

The following guidelines highlight the important laboratory biosafety aspects for all three phases of laboratory processes.

How to cite this article: Bhat $\mathrm{V}$, Chavan $\mathrm{P}$, Biswas $\mathrm{S}$, Gupta S, Khattry N, Thakkar P. Laboratory biosafety issues related to coronavirus disease 2019. Indian J Med Paediatr Oncol 2020;41:450-3.

\section{Vivek Bhat ${ }^{1}$, Preeti Chavan², Sanjay Biswas ${ }^{1}$, Sudeep Gupta ${ }^{3}$, Navin Khattry ${ }^{3}$, Prafulla Thakkar ${ }^{4}$}

${ }^{1}$ Department of Microbiology, Tata Memorial Centre, Navi Mumbai, Maharashtra, India, ${ }^{2}$ OIC, Composite Laboratory, ACTREC-Tata Memorial Centre, Navi Mumbai, Maharashtra, India, ${ }^{3}$ Department of Medical Oncology, Tata Memorial Centre, Navi Mumbai, Maharashtra, India, ${ }^{4}$ Department of General Medicine, ACTREC-Tata Memorial Centre, Navi Mumbai, Maharashtra, India

Submitted: 20 -Apr-2020 Revised: 14-May-2020 Accepted: 21-Jun-2020 Published: 29-Aug-2020

Address for correspondence: Dr. Preeti Chavan, OIC, Composite Laboratory, ACTREC-Tata Memorial Centre, Sector: 22, Kharghar, Navi Mumbai - 410 210, Maharashtra, India.

E-mail: drprreeti@yahoo.com

Access this article online

Website: www.ijmpo.org

DOI: 10.4103/ijmpo.ijmpo_176_20 Quick Response Code:

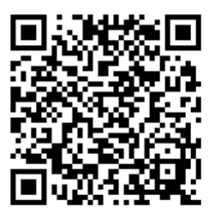




\section{Preanalytical Phase}

\section{Sample collection}

Timely and effective communication between clinicians and laboratory personnel is of utmost importance so that the risk incurred in handling these specimens from patients with either suspected or confirmed COVID-19 infection is minimized. Other than respiratory secretions including sputum, nasopharyngeal secretions, and bronchoalveolar lavage fluid, the presence of COVID-19 virus has been observed in blood (1\%) and feces (29\%). ${ }^{[7]}$ Standard precautions must be followed at all times when laboratory staff handle clinical specimens that may contain infectious microorganism. Standard precautions must include hand hygiene along with the use of personal protective equipment (PPE), such as N95 masks, gloves, gowns, and eye protection [Table 1].

All samples are collected in appropriate containers and all containers are decontaminated by $62 \%-71 \%$ ethanol (alcohol) before transporting them to the laboratory. Specimens are appropriately labeled and the laboratory alerted about the same. These instructions are for all the laboratory specimens including hematology, biochemistry, histopathology, and microbiology.

This will ensure proper specimen handling at all levels.

\section{Sample packaging and transport}

The WHO has advised all viral cultures or isolates should be designated as category A infectious substance (UN 2814). This includes infectious substances meeting the criteria which cause disease in humans or both in humans and animals. All other biochemical and hematology samples are designated as biological substance category B (UN 3373).

All samples are transported using universal precautions. The packaging used is triple packaging. It consists of the primary tube which is covered with an absorbent material and is placed in the secondary receptacle which is leak-proof. This is placed in the transportation box with biohazard symbol. The sample is carried in upright position.

The required temperature of the sample in transport is maintained by the use of dry ice or gel packs. ${ }^{[8]}$ Histopathology samples are transported in containers with formalin. The samples are transported to the laboratory as soon as possible along with the appropriate documentation. The staff handling the samples should be trained and use PPE and practice hand hygiene.

Special care should be taken while transporting samples for the detection of the virus which are collected in viral transport medium with a swab from nasopharyngeal/nasal and oropharyngeal sites. These samples should not be delivered through pneumatic chutes.

\section{Sample receipt and processing}

Upon receipt of samples in the laboratory, all samples are checked for appropriateness of the samples and documentation. Duly filled requisition/request forms accompany the samples. Care must be taken to ensure the forms or the outside of container are not soiled. Samples should be handled only by trained staff using PPE and taking universal precautions. All samples, including those for molecular testing, should undergo initial processing in a biosafety cabinet (BSC) as recommended by the WHO. ${ }^{[9]}$ Samples are centrifuged only in sealed tubes to avoid aerosol formation, and only closed centrifugation must be performed If the samples need to be separated in smaller tubes or need to be aliquoted, the procedure should be undertaken only in a BSC. This is applicable to all serology, biochemistry, cytopathology, and hematology specimens.

Grossing of all histopathology specimen is done only after wearing full PPE. Only formalin-fixed specimens should be grossed. Fresh specimen processing such as for frozen section should be avoided. If it is necessary, full PPE should be worn before processing frozen section. Immediately after the procedure, the specimen is fixed in formalin. Extra precautions may be taken while using saw for bony specimens which can produce aerosols. Use of face shields and/or goggles, masks, and gowns is a must for processing these specimens. The counters should be cleaned with disinfectant between the two specimens.

\section{Analytical Phase}

The following laboratory procedures can be handled in a biosafety level 2 facility using standard precautions ${ }^{[10]}$ [Table 1]:

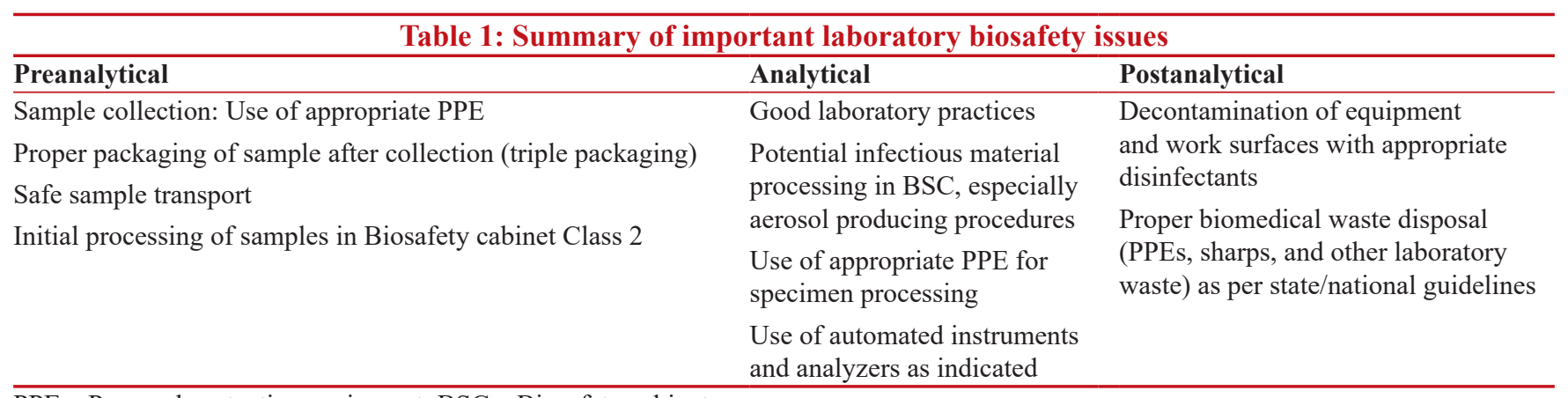

PPE - Personal protective equipment; BSC - Biosafety cabinet 
- All nonpropagatory routine diagnostic laboratory work

- Automated instruments and analyzers for all routine hematology and clinical chemistry tests

- All staining and microscopic examination of smears that are fixed

- Processing of routine bacterial culture and susceptibility testing

- Histopathologic examination and processing of tissues fixed in formalin

- Molecular tests including extraction of nucleic acid from patient specimen

- Triple packaging of specimens to be transported to referral laboratories for further testing (specimens collected in a sealed primary container that has been decontaminated).

Good laboratory practices and procedures should be followed at all times. This includes prohibition of eating food, drinking, smoking, and applying cosmetics in the working areas of the laboratory by the staff. Only adequately trained staff should be posted for handling and processing of samples. Appropriate PPE such as gloves, masks, gown, goggles, and face shields should be worn as and when required by the staff.

It should be ensured while undertaking all technical procedures that there is minimal formation of aerosols and droplets. All procedures that may require manipulations of potentially infectious materials and may result in splashing, droplet, or aerosols of infectious material should be performed in BSC-Class 2 that are appropriately maintained and validated. Such procedures may include but are not limited to loading and/or unloading of sealed centrifuge cups, opening of vials containing infectious materials, grinding, vortexing, vigorous shaking and mixing of samples, sonic disruptions of samples, etc., Use of needles should generally be avoided or restricted. Mouth pipetting is not to be allowed in the laboratory.

\section{Postanalytical Phase}

This includes reporting of results as well disposal of biomedical waste [Table 1].

All reports are released only by the authorized personnel.

All contaminated sharp should be disposed in containers that are puncture-proof, fitted with lids, and are treated as infectious waste. It is the responsibility of the laboratory administration to provide adequate biohazard containers in the immediate working areas. All biomedical waste should be disposed as per state and national guidelines. The samples are preserved postanalysis as per laboratory criteria and are disposed using national/state biomedical waste guidelines.

Strict hand hygiene routine should be practiced after handling infectious material, before exiting the laboratory working area and before having food, by all staff working in the laboratory. All the PPE used should be removed and disposed as infectious waste before leaving the laboratory.

\section{Decontamination}

There are no definite guidelines for the decontamination practices for this novel virus. However, it is recommended to use disinfectants that are found to be effective against similar enveloped viruses such as Middle East respiratory syndrome coronavirus. ${ }^{[11]}$ This includes use of $1 \%$ sodium hypochlorite, $62 \%-71 \%$ ethanol for surface disinfection or hydrogen peroxide $(0.5 \%)$. All surfaces should be disinfected frequently. It is important to ensure appropriate contact time as well as dilutions and expiry date for prepared disinfectants as advised by the manufacturers.

\section{Laboratory Waste Management}

All PPE and other laboratory waste are to be collected in yellow bags (as per state pollution control board) and autoclaved at $121^{\circ} \mathrm{C}$ and $15 \mathrm{psi}$ for $60 \mathrm{~min}$ using chemical and/or biological indicators. It is then labeled as COVID-19 waste before handing over to certified biomedical waste treatment facility in double-layered yellow bags. All waste handlers must wear adequate PPE, when disposing of COVID-19 waste. The laboratory should follow any additional state or national guidelines on biomedical waste disposal.

\section{Incident Reporting}

All staff should be trained to report and handle adverse incidents such exposure to infectious materials, spills, etc., The laboratory should have processes for reporting of adverse incidents. Spill management kits should be available in all areas of the laboratory along with procedures to manage the spills. First-aid kits should be available to staff.

It is thus important to ensure that the above-mentioned laboratory biosafety measures be implemented and adhered to minimize the risk of laboratory transmission of COVID-19 to health-care workers.

\section{Financial support and sponsorship}

Nil.

\section{Conflicts of interest}

There are no conflicts of interest.

\section{References}

1. Zhu N, Zhang D, Wang W, Li X, Yang B,Song J, et al. A novel coronavirus from patients with 243 pneumonia in China, 2019. N Engl J Med 2020;382:727-33.

2. Available from: https://www.who.int/emergencies/diseases/ novelcoronavirus-2019/events-as-they-happen. 3. [Last accessed on 2020 Apr 14].

3. WHO Infection Prevention and Control Guidance for COVID-19. Available from: https://www.who.int/emergencies/diseases/ 
novelcoronavirus-2019/technical-guidance/infection-preventionandcontrol. [Last accessed on 2020 Apr 14].

4. Interim Infection Prevention and Control Recommendations for Patients with Suspected or Confirmed Coronavirus Disease 2019 (COVID-19) in Healthcare Settings. Available from: https://www.cdc.gov/coronavirus/2019-ncov/infectioncontrol/controlrecommendations.html 5. [Last accessed on 2020 Apr 15].

5. Infection Prevention and Control for COVID-19 in Healthcare Settings. Available from: https://www.ecdc.europa.eu/en/ publications-data/infection-prevention-and-control-covid19healthcare-settings. [Last accessed on 2020 Apr 15].

6. Section 1: Introduction. In: Chosewood C, Deborah E, editors. Biosafety in Microbiological and Biomedical Laboratories, $5^{\text {th }}$ ed. HHS Publication No. (CDC) 21-1112; 2009. p. 1.

7. Zhu N, Zhang D, Wang W, Li X, Yang B, Song J, et al. A novel coronavirus from patients with 243 pneumonia in China, 2019. N Engl J Med 2020;382:727-33.

8. WHO/CDS/EPR/2007.2 Guidance on Regulations for the Transport of Infectious Substances; 2007.

9. Available from: http://file://C:/Users/admin/Downloads/ WHOWPE-GIH-2020.2-eng.pdf.10. [Last accessed on 2020 Apr 16].

10. Available from: https://www.cdc.gov/coronavirus/2019-ncov/lab/ lab-biosafety-guidelines.html. [Last accessed on 2020 Apr 15].

11. World Health Organisation. Laboratory Biosafety Guidance Related to Coronavirus Disease (COVD-19): Interim Guidance; 2020. p. 2. 